\title{
Spectrophotometric Determination of Europium, Terbium and Yttrium in a Perchloric Acid Solution by the Kalman Filter Approach
}

\author{
Yongnian $\mathrm{NI}^{\dagger}$ and Yingliang $\mathrm{WU}$ \\ Department of Chemistry, Nanchang University, Jiangxi, Nanchang 330047, China
}

\begin{abstract}
In the presence of perchloric acid, rare-earth elements of europium, terbium and yttrium at large concentrations have well-shaped absorption spectra in the ultraviolet region even without the addition of a chromogenic reagent. However, it is difficult to determine each of them individually, because they have serious overlapping peaks. In this study, a chemometric method, Kalman filter, was used to resolve the overlapped spectra and to determine the individual components. In contrast to conventional implementation of the Kalman filter, an absorption-coefficient matrix derived from a set of mixtures of known composition by multiple linear regression (MLR) was found to yield better results in this work. For a set of synthetic mixtures of europium, terbium and yttrium, their molar absorptivities were determined to be 1945, 3756 and 1446, respectively. The proposed method was also applied to the determination of europium, terbium and yttrium in a sample of phosphor powder product; a satisfactory result was obtained.
\end{abstract}

Keywords Kalman filter, spectrophotometry, europium, terbium and yttrium

Natural deposits of rare-earth elements are abundant in China, and the rare-earth industry is important in the Chinese economy, because of their special photogenic, magnetic, mechanical and nuclear properties; rare-earth elements are widely applied in the fields of glass and ceramic industries, metallurgy, electronics, agriculture and natural science. ${ }^{1}$ Chemists are increasingly paying attention to studies of the environmental, medical and biological applications of rare-earth elements to develop a rare-earth industry. Therefore, methods for their rapid, sensitive and accurate determination and separation are of great importance. However, it is extremely difficult to determine them individually without a preseparation due to the similarity of their chemical properties. More selective techniques, such as mass spectrometry (MS), inductively coupled plasma atomic emission spectrometry (ICP-AES), and inductively coupled plasma mass spectrometry (ICP-MS) and chromatography, are generally applied to individual determination of rare-earth elements. Although satisfactory results have been obtained, they have the disadvantages of relatively high costs incurred, and sometimes serious base effects. ${ }^{2}$ Therefore, analysts have done much work to find some convenient methods for the individual quantification of these rare-earth species. Recently, spectrophotometry combined with chemometric approaches are often applied to the quantitative analysis of mixtures of rareearth elements. ${ }^{3-7}$ However, many of these reported studies just involve the trace analysis of rare-earth elements, and generally a color reaction with the addition of a chromogenic reagent is necessary. For the determination of a mixture of rare-earth elements with major concentrations, a complexometric titration method can generally be used; it is only possible to determine the total amount of rare-earth elements. ${ }^{8}$

It has been found that in perchloric acid, some rare earth elements have well-shaped absorption spectra in the ultraviolet region without the addition of any chromogenic reagents, though these spectra are seriously overlapped. In these cases, chemometric approaches have the ability to resolve and quantify mixtures of rare-earth elements. Recently, Peralta-Zamora et al. ${ }^{9}$ have reported a method to determine lanthanide mixtures by partial least squares (PLS); the molar absorptivities obtained for these lanthanides are in the range of $10.49-26.00$.

In the present work, it was found that heavy rareearth elements (europium, terbium and yttrium) with high-level concentrations have good adsorption spectra in $1.0 \mathrm{~mol} \mathrm{l}^{-1}$ perchloric acid with strongly overlapped spectra in the ultraviolet region. A Kalman filter was used to resolve the spectra of the mixture. In contrast to conventional implementation of the Kalman filter, an improved Kalman filter using the absorption-coefficient matrix obtained from a known set of mixtures by multiple linear regression (MLR) was found to yield the best results in this work. 


\section{Experimental}

\section{Reagents and apparatus}

Solutions of europium, terbium and yttrium were prepared from their oxides, about $0.2 \mathrm{~g}$ of the oxide was weighted and introduced into a platinum crucible, then heated at $850^{\circ} \mathrm{C}$ in a muffle furnace for one hour to digest any organic matter. After cooling, the sample was transferred into a $100 \mathrm{ml}$ flask and $5.0 \mathrm{ml}$ of concentrated hydrochloric acid was added; the solution was warmed until white fumes of un-reacting hydrochloric acid appeared and the sample was almost dry. The residue was then transferred to a $100 \mathrm{ml}$ volumetric flask and dissolved by distilled water and diluted to the mark. All of these solutions were standardized by complexometric titration with EDTA using Xylenol Orange as an indicator. The concentrations of europium, terbium and yttrium with respect to their oxides were $2.036,2.040$ and $2.030 \mathrm{~g} \mathrm{l}^{-1}$, respectively. The medium solution of $1.0 \mathrm{~mol} \mathrm{l}^{-1}$ of perchloric acid was prepared according to the classical method. All of the reagents used in this work were of analytical reagent grade.

Spectrophotometric measurements were made on a Hitachi U-2000 double-beam spectrophotometer. The $\mathrm{pH}$ of the solutions was measured with a Model SA 720 $\mathrm{pH}$ meter (Orion). The computations were processed by a 586 computer. All of the programs used in the computing process were written in MATLAB 4.2C. All of the experiments were carried out at $25^{\circ} \mathrm{C}$.

\section{Procedure}

A suitable amount of a sample solution of rare-earth elements or their mixture was pipetted into a $10 \mathrm{ml}$ volumetric flask and diluted with $1.0 \mathrm{~mol} \mathrm{l}^{-1}$ perchloric acid to the mark. The absorbance of this solution was measured in $1.0 \mathrm{~cm}$ cuvettes between 191 and $235 \mathrm{~nm}$ at $2 \mathrm{~nm}$ intervals with respect to a reagent blank of 1.0 mol $1^{-1}$ perchloric acid. The absorbance data of unknown samples obtained from the experiments were treated by a chemometric approach, Kalman filter; the concentration of each rare-earth element was obtained.

\section{Results and Discussion}

\section{Selection of the medium}

In this work, several materials, such as hydrochloric acid, acetylacetone, EDTA and perchloric acid, were investigated as media for spectrophotometric measurements. It was found that the perchloric acid gives the more sensitive, stable and reproducible absorption spectra than the other materials. Figure 1 shows the absorbance spectra of europium, terbium and yttrium in perchloric acid with strongly overlapping waves. There are no distinguished differences between the wavelengths givng the maximum absorbance of these three rare-earth elments (190.6, 190.6 and $190.9 \mathrm{~nm}$ for

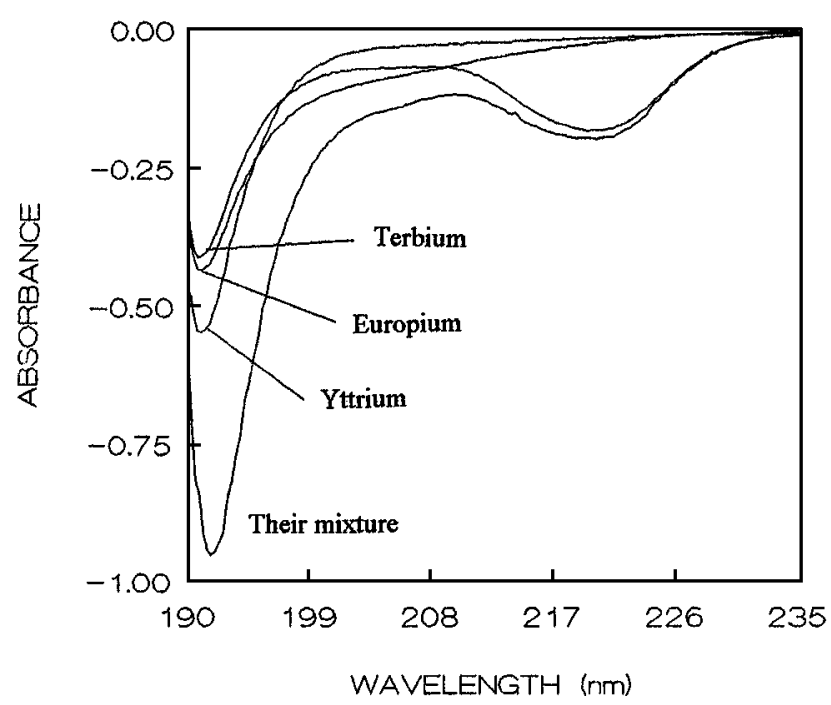

Fig. 1 Absorption spectra of each rare-earth element in 1.0 mol 1-1 perchloric acid. $C_{\mathrm{Y}}=81.2 \mathrm{mg} \mathrm{l}^{-1}, C_{\mathrm{Eu}}=81.4 \mathrm{mg} \mathrm{l}^{-1}$ and $C_{\mathrm{Tb}}=81.6 \mathrm{mg} \mathrm{l}^{-1}$, with respect to their oxide.

europium, terbium and yttrium, respectively), but there are obvious differences between their wave shapes, which make their simultaneous determination possible. The molar absorptivities of europium, terbium and yttrium are 1945, 3756 and 1446, respectively, which are bigger than those found in the literature. ${ }^{9}$ Furthermore, it should be noted that the absorbance of all these three rare-earth elements is negative in perchloric acid, due to complexations with perchloric acid.

From Fig. 1, it can be found that not only the additivity of the absorbance of this system is poor, especially near to the maximum absorption peaks, but also the peak position of the mixture sample is slightly shifted as compared with those of individual samples. All of these are caused by the non-linearity and interaction between the compounds.

\section{Kalman filter and calculation of the absorption coefficient matrix}

The kalman filter method has been available for several decades and has been applied to a large number of different problems, mostly in the area of control theory. However, it has also been applied to quantitative chemical problems. Most of these applications were concerned with multicomponent problems, including spectrophotometric and electrochemical systems. ${ }^{10-15}$ Their studies have demonstrated that the Kalman filter method is an efficient approach to resolve multicomponent systems with overlapping peaks.

Details of the Kalman filter method can be found elsewhere. ${ }^{16,17}$ In this section, only the essential details are described. The Kalman filter relies on a linear model consisting of two equations: one to describe the chemical system and the other to describe the measurement process. The first of these equations, the system model, can be expressed as 


$$
\boldsymbol{x}_{k+1}=\boldsymbol{F}_{k} \boldsymbol{x}_{k}+\boldsymbol{w}_{k}
$$

where $\boldsymbol{x}_{k}$ is a $n \times 1$ state vector of the concentrations at point $k, \boldsymbol{F}_{k}$ is a $n \times n$ state transition matrix that relates $\boldsymbol{x}_{k}$ to $\boldsymbol{x}_{k+1}$, and $\boldsymbol{w}_{k}$ is a $n \times 1$ vector describing noise processes present. Because the concentration of the $n$ component does not change as the spectra is scanned from one wavelength to the next, and the "mode noise", $\boldsymbol{w}_{k}$, can be taken as a zero vector, Eq.(1) simply reduces to

$$
\boldsymbol{x}_{k+1}=\boldsymbol{I} \boldsymbol{x}_{k}
$$

where $\boldsymbol{I}$ is the identity matrix.

The following equation describes the measurement model:

$$
y_{k}=\boldsymbol{h}_{k}^{\mathrm{T}} \boldsymbol{x}_{k}+v_{k},
$$

where $\boldsymbol{h}_{k}$ is the $k$ th column vector of the $n \times m$ absorption-coefficient matrix $\boldsymbol{H}, y_{k}$ is the measured absorbance at point $k$ and $v_{k}$ is the noise contribution to the measurement at the step $k$. It is assumed that $v_{k}$ is a zero-mean white-noise, which is associated with a known variance, $r_{k}$. In this system, because the variance, $r_{k}$, remains constant, the subscript $k$ can be neglected.

The Kalman filter is carried out recursively; as each new data point is measured, the concentration vector, $\boldsymbol{x}_{k-1}$, from the previous step is updated by the new measurement, $y_{k}$, to yield an improved estimate, $\boldsymbol{x}_{k}$, in such a way as to minimize the estimation error, $e_{k}$ :

$$
\boldsymbol{e}_{k}=\boldsymbol{x}_{k}-\boldsymbol{x}_{k-1},
$$

and its associated $n \times n$ error covariance matrix $\boldsymbol{P}_{k}$ (i.e., $\left.\boldsymbol{P}_{k}=\boldsymbol{e}_{k} \boldsymbol{e}_{k}^{\mathrm{T}}\right)$. The first step in the Kalman filter algorithm is a calculation of the $n \times 1$ matrix of Kalman gain vectors, $\boldsymbol{K}_{k}$ :

$$
\boldsymbol{K}_{k}=\boldsymbol{P}_{k} \boldsymbol{h}_{k}\left(\boldsymbol{h}_{k}^{\mathrm{T}} \boldsymbol{P}_{k} \boldsymbol{h}_{k}+r\right)^{-1},
$$

which optimally minimizes the terms in the error covariance matrix, $\boldsymbol{P}_{k}$, in a least-squares sense. Next, the Kalman gain factors, $\boldsymbol{K}_{k}$, are used to blend together the prior estimates, $\boldsymbol{x}_{k-1}$, and the new measurement, $y_{k}$, to yield an improved estimate of $\boldsymbol{x}_{k}$,

$$
\boldsymbol{x}_{k}=\boldsymbol{x}_{k-1}+\boldsymbol{K}_{k}\left(y_{k}-\boldsymbol{h}_{k}^{\mathrm{T}} \boldsymbol{x}_{k-1}\right) \text {. }
$$

This allows the error covariance matrix to be updated,

$$
\boldsymbol{P}_{k+1}=\boldsymbol{P}_{k}-\boldsymbol{K}_{k} \boldsymbol{h}_{k}^{\mathrm{T}} \boldsymbol{P}_{k}
$$

Equations (5) - (7)are then repeated using the latest estimates of $\boldsymbol{x}_{k}$ and $\boldsymbol{P}_{k+1}$, until there are no more measurement data, $y_{k}$, to process, or until successive sequences of $\boldsymbol{x}_{k}$ converge. In order to initiate the recursive procedure, initial estimates of $\boldsymbol{x}_{0}$ and $\boldsymbol{P}_{0}$ are required; $\boldsymbol{x}_{0}$ is generally set to zero and the initial error covariance matrix $\boldsymbol{P}_{0}$ is set to

$$
\boldsymbol{P}_{0}=\alpha^{2}\left(r / \boldsymbol{h}_{1}^{\mathrm{T}} \boldsymbol{h}_{1}\right) \boldsymbol{I}
$$

where $\alpha$ is a factor which is related to the precision, and is generally selected to be $10-100 ; r$ is selected to be $10^{-6}$ and $\boldsymbol{I}$ is an $n \times n$ identity matrix.

An important consideration in the application of the Kalman filter for multicomponent analysis is an accurate deduction of the absorption-coefficient matrix, $\boldsymbol{H}$. It is highly desirable to determine the absorption coefficient matrix under exactly the same experimental condition as is used for the sample solutions. Generally, $\boldsymbol{H}$ is obtained from the single-component curves (responses) that have been normalized by dividing by their corresponding concentrations. In this work, three methods were used to calculate $\boldsymbol{H}$ : (i) the aforementioned conventional method, (ii) a multiple linear regression (MLR) model, in which a set of multicomponent standards of known composition are used, together with the following equations:

$$
\begin{aligned}
& \boldsymbol{Y}_{\text {known }}=\boldsymbol{H}^{\mathrm{T}} \boldsymbol{X}_{\text {known }} \\
& \boldsymbol{H}^{\mathrm{T}}=\boldsymbol{Y}_{\text {known }} \boldsymbol{X}_{\text {known }}^{\mathrm{T}}\left(\boldsymbol{X}_{\text {known }} \boldsymbol{X}_{\text {known }}^{\mathrm{T}}\right)^{-1}
\end{aligned}
$$

where, $\boldsymbol{X}_{\text {known }}$ and $\boldsymbol{Y}_{\text {known }}$ represent the concentration matrix and measured absorption matrix for the multicomponent standards, respectively. In this work an orthogonally designed set of nine multicomponent standards ${ }^{18,19}$, denoted by $\mathrm{OA}_{9}\left(3^{4}\right)$, which contains components in the concentration range $20-80 \mathrm{mg} \mathrm{l}^{-1}$, were used; also, (iii) is similar to (ii), except that a non-zero intercept was introduced into the coefficient matrix $\boldsymbol{H}$ to correct for any non linearity or interaction between the components. ${ }^{20}$

\section{Determination of synthetic samples}

In this work, the quantitative analysis of nine synthetic mixtures of rare-earth element absorption-coefficient matrices obtained by different methods was investigated; the results are listed in Table 1. The Kalman filter is a discrete linear filter. If the linearity and the additivity of the analytical system are both good, precise filtering results can be obtained by using the traditional Kalman filter with the absorption-coefficient matrix obtained directly from the absorption spectra of each single component, even if there is a seriously overlapped spectra. On the contrary, it is difficult to obtain precise results if the linear relationship of the system is poor. It should be noted that, in this work, the most precise results were obtained by applying the MLR method to estimate the absorption-coefficient matrix with a non-zero intercept (see Table 1).

\section{Determination of europium, terbium and yttrium in sample of phosphor powder}

A sample $(0.2010 \mathrm{~g})$ of phosphor powder (since the content of terbium in this real sample is small, so a suit- 
Table 1 Determination results of synthetic mixtures of europium, terbium and yttrium by use of different Kalman filter methods

\begin{tabular}{|c|c|c|c|c|c|c|c|c|c|c|c|c|}
\hline \multirow{3}{*}{$\begin{array}{l}\text { Sample } \\
\text { number }\end{array}$} & & & & \multicolumn{9}{|c|}{ Found $(\mathrm{mg} / 10 \mathrm{ml})$} \\
\hline & \multicolumn{3}{|c|}{ Added $(\mathrm{mg} / 10 \mathrm{ml})$} & \multicolumn{3}{|c|}{ Method $1^{a}$} & \multicolumn{3}{|c|}{ Method $2^{b}$} & \multicolumn{3}{|c|}{ Method $3^{c}$} \\
\hline & $\mathrm{Y}_{2} \mathrm{O}_{3}$ & $\mathrm{Eu}_{2} \mathrm{O}_{3}$ & $\mathrm{~Tb}_{4} \mathrm{O}_{7}$ & $\mathrm{Y}_{2} \mathrm{O}_{3}$ & $\mathrm{Eu}_{2} \mathrm{O}_{3}$ & $\mathrm{~Tb}_{4} \mathrm{O}_{7}$ & $\mathrm{Y}_{2} \mathrm{O}_{3}$ & $\mathrm{Eu}_{2} \mathrm{O}_{3}$ & $\mathrm{~Tb}_{4} \mathrm{O}_{7}$ & $\mathrm{Y}_{2} \mathrm{O}_{3}$ & $\mathrm{Eu}_{2} \mathrm{O}_{3}$ & $\mathrm{~Tb}_{4} \mathrm{O}_{7}$ \\
\hline 1 & 0.3045 & 0.5090 & 0.7140 & 0.2436 & 0.4500 & 0.6732 & 0.3045 & 0.4724 & 0.7038 & 0.3045 & 0.4947 & 0.7222 \\
\hline 2 & 0.5075 & 0.5090 & 0.5100 & 0.4669 & 0.5497 & 0.4692 & 0.5481 & 0.5497 & 0.4488 & 0.5287 & 0.5395 & 0.4916 \\
\hline 3 & 0.7105 & 0.5090 & 0.3060 & 0.6699 & 0.4683 & 0.2611 & 0.7308 & 0.5294 & 0.2815 & 0.6943 & 0.5497 & 0.2978 \\
\hline 4 & 0.3045 & 0.3054 & 0.5100 & 0.2233 & 0.3359 & 0.5345 & 0.2700 & 0.2688 & 0.5202 & 0.2842 & 0.2810 & 0.5304 \\
\hline 5 & 0.5075 & 0.3054 & 0.3060 & 0.4466 & 0.3359 & 0.2734 & 0.4872 & 0.3258 & 0.2856 & 0.5116 & 0.3054 & 0.2876 \\
\hline 6 & 0.7105 & 0.3054 & 0.7140 & 0.6293 & 0.3319 & 0.6895 & 0.6821 & 0.2850 & 0.6936 & 0.6902 & 0.3095 & 0.7099 \\
\hline 7 & 0.3045 & 0.7126 & 0.3060 & 0.2639 & 0.6922 & 0.2611 & 0.2903 & 0.6983 & 0.2856 & 0.3045 & 0.7024 & 0.2938 \\
\hline 8 & 0.5075 & 0.7126 & 0.7140 & 0.4263 & 0.7635 & 0.6691 & 0.4872 & 0.6719 & 0.6528 & 0.5075 & 0.7024 & 0.6997 \\
\hline 9 & 0.7105 & 0.7126 & 0.5100 & 0.6581 & 0.7741 & 0.4611 & 0.7241 & 0.6665 & 0.4912 & 0.7087 & 0.7112 & 0.5056 \\
\hline $\begin{array}{l}\mathrm{RPE}_{S}{ }^{d} \\
\mathrm{RPE}_{T}\end{array}$ & & & & $\begin{array}{l}11.66 \\
9.17\end{array}$ & 7.91 & 7.36 & $\begin{array}{l}4.52 \\
5.65\end{array}$ & 6.09 & 6.18 & $\begin{array}{l}2.46 \\
2.96\end{array}$ & 3.74 & 2.50 \\
\hline
\end{tabular}

a. Absorption coefficient matrix obtained by conventional method was used. b. Absorption coefficient matrix without non-zero intercept obtained by MLR method was used. c. Absorption coefficient matrix with non-zero intercept obtained by MLR method was used. d. $\mathrm{RPE}_{\mathrm{S}}$ and $\mathrm{RPE}_{\mathrm{T}}$ are the relative prediction errors for single component and total components, respectively. ${ }^{21}$

Table 2 Determination of rare-earth elements in a sample of phosphor powder $\left(\mathrm{mg} \mathrm{g}^{-1}\right)^{\mathrm{a}}$

\begin{tabular}{lccc}
\hline \multicolumn{1}{c}{ Method } & $\mathrm{Eu}_{2} \mathrm{O}_{3}$ & $\mathrm{~Tb}_{4} \mathrm{O}_{7}$ & $\mathrm{Y}_{2} \mathrm{O}_{3}$ \\
\hline Kalman filter & 48.1 & 41.3 & 902.0 \\
ICP-AES & 43.5 & 40.9 & 901.5 \\
\hline
\end{tabular}

a. The sample was obtained from Jiangyin Advanced Materials Resources Co. Ltd., China.

b. Method 3 in Table 1 was used.

able amount of terbium oxide was added) was weighted and transferred into a $100 \mathrm{ml}$ flask. $5.0 \mathrm{ml}$ of concentrated hydrochloric acid was added to the flask and the sample was dissolved by warming until the white fumes of hydrochloric acid appeared and let the reaction completely finished. After cooling, the residue was dissolved and transferred in to a $100 \mathrm{ml}$ volumetric flask with distilled water and diluted to the mark. Then, $2.0 \mathrm{ml}$ of this sample solution was taken for a determination using the general procedure described previously. From the results listed in Table 2, it can be seen that, as compared with the ICP-AES method, precise results are obtained with the introduction of the non-zero intercept into the calibration procedure for the Kalman filter.

It should be noted that the amount of yttrium in this real sample is too large compared with the compositions of the standard samples used to evaluate the coefficient matrix, $\boldsymbol{H}$. If a classical multivariate calibration method, such as classical least squares ${ }^{20,22}$ is applied to analyze this sample, no satisfactory results will be obtained, because the unstable data near to the peaks will be used for calculation. However, different from the classical least-squares method, the Kalman filter is a recursive method. In this work, a filter calculation was carried out by using the data in sequence from wavelength 235 to $190 \mathrm{~nm}$, and generally, the calculation is converged before $200 \mathrm{~nm}$, so only a part of the data is used in Kalman filter performance. It can be seen that although the content of yttrium is larger, its contribution to the absorbance of the mixture is very small in this wavelength region and the absorption coefficient of yttrium obtained from above standard samples is effective for filtering. Thus in this work, it is still possible to obtain satisfied results for all of these three rare-earth elements by using the Kalman filter approach.

The Kalman filter can be applied to resolve seriously overlapping spectra of mixtures of europium, terbium and yttrium. Furthermore, the results can be improved by the use of the absorption-coefficient matrix with a non-zero intercept obtained from a set of mixtures of known composition by a multiple linear-regression method, since this method can reduce or eliminate the effect of any non-linearity and any interaction between the components.

We are grateful to the Jiangxi Province Natural Science Foundation (JXNSF, No. 96206) and the National Natural Science Foundation of China (NSFC, No. 29765001) for financial support.

\section{References}

1. G. Xu, "Rare Earth (in Chinese)", Metallurgical Industry Press, Beijing, 1995.

2. K. W. Jackson and T. M. Mahmood, Anal. Chem., 66, 252R (1994).

3. J. M. Garcia Fraga, A. I. Jimenez Abizanda, F. Jimenez Moreno, J. J. Arias Leon and J. Havel, Microchem. J., 54, 32 (1996).

4. Z. Wang and G. Chen, Microchem. J., 53, 122 (1996).

5. L. Jancar, J. Preisler and L. Sommer, Collect. Czech. Chem. Commun., 58, 1509 (1993).

6. R. D. Bautista, A. I. Jimenez, F. Jimenez and J. J. Arias, Talanta, 43, 421 (1996).

7. H. Wang, D. Wang, Y. Wang, S. Chen and F. Zhang, 
Analyst [London], 120, 1603 (1995).

8. Y. Chen, "Complexometric Titrations (in Chinese)", Chinese Science Press, Beijing, 1986.

9. P. Peralta-Zamora, L. Cornejo-Ponce, N. Nagata and R. J. Poppi, Talanta, 44, 1815 (1997).

10. H. N. J. Poulisse, Anal. Chim. Acta, 112, 361 (1979)

11. H. N. J. Poulisse and P. Engelen, Anal. Lett., 13, 1211 (1980).

12. T. F. Brown and S. D. Brown, Anal. Chem., 53, 1410 (1981).

13. C. A. Scolari and S. D. Brown, Anal. Chim. Acta, 166, 253 (1984).

14. C. A. Scolari and S. D. Brown, Anal. Chim. Acta, 178, 239 (1985).

15. Y. Ni, M. Selby, S. Kokot and M. Hodgkinson, Analyst
[London], 118, 1049 (1993).

16. R. E. Kalman, J. Basic Eng., 82, 34 (1960).

17. S. D. Brown, Anal. Chim. Acta, 181, 1 (1986).

18. Mathematics Department of Beijing University, "Orthogonal Design (in Chinese)", Beijing University Press, Beijing, 1976.

19. Y. Ni, J. Bai and L. Jin, Anal. Chim. Acta, 329, 65 (1996).

20. C. W. Brown, P. F. Lynch, R. J. Obremski and D. S Lavery, Anal. Chem., 54, 1472 (1982).

21. M. Otto and W. Wegscheider, Anal. Chem., 57, 63 (1985).

22. Y. Iida, K. Goto, M. Furukawa and S. Shibata, Bunseki Kagaku, 32, 401 (1983).

(Received February 15, 1999) (Accepted August 26, 1999) 